Article

\title{
Possibility Neutrosophic Cubic Sets and Their Application to Multiple Attribute Decision Making
}

\author{
Huiling Xue ${ }^{1}\left(\mathbb{D}\right.$, Xiaotong Yang $^{2}$ and Chunfang Chen ${ }^{2, *}$ (1) \\ 1 School of Economics, Jinan University, Guangzhou 510632, Guangdong, China; huilingxue0116@163.com \\ 2 Department of Mathematics, Nanchang University, Nanchang 330031, Jiangxi, China; \\ yangxt19940509@163.com \\ * Correspondence: ccfygd@sina.com
}

Received: 4 January 2020 ; Accepted: 4 February 2020; Published: 10 February 2020

\begin{abstract}
The neutrosophic cubic sets are an extension of the cubic sets to the neutrosophic sets. It contains three variables, which respectively represent the membership degree, non-membership degree and uncertainty of the element to the set. The score function is an important indicator in the multi-attribute decision-making problem. In this paper, we consider the possibility that an element belongs to a set and put forward the concept of possibility neutrosophic cubic sets. On this basis, we introduce some related concepts and give the binary operation of possibility neutrosophic cubic sets and use specific examples to supplement the corresponding definition. Meanwhile, a decision-making method based on the score function of possibility neutrosophic cubic sets is proposed and a numerical example is given to illustrate the effectiveness of the proposed method.
\end{abstract}

Keywords: possibility neutrosophic cubic sets; score function; multiple attribute decision making

\section{Introduction}

Many practical problems in life are uncertain, such as medical diagnosis, project site selection, economic activities and so on. Therefore, some scholars have put forward many new theories to solve these practical problems. In 1965, Zadeh [1] first proposed the fuzzy sets (FSs), breaking the idea of classical set theory, thinking that things have a certain degree of ambiguity, and using ambiguities can more accurately express many difficult-to-describe problems in real life. The membership function is the basis of fuzzy set theory and a generalization of classical set theory. It shows the degree of membership of an element to a set. The value of the membership function is in the interval $[0,1]$. The closer the value is to 1 , it indicates the degree of membership is greater. Generally, what we call fuzzy sets is more abstract, but the membership of fuzzy sets is a specific real number; therefore, fuzzy sets have significant advantages in real life and have been widely used [2-5]. Based on the advantages of fuzzy sets, there has been a lot of studies since then which have extended the research on fuzzy sets [6-9]. Due to the complexity of the problem, the fuzzy set theory presents some shortcomings in the process of solving the problem. Therefore, some researchers put forward various extended forms of fuzzy sets, such as intuitionistic fuzzy sets (IFSs) [10], interval valued intuitionistic fuzzy sets (IVIFSs) [11], hesitant fuzzy sets (HFSs) [12], and various extension sets of fuzzy sets have been used to solve many uncertain problems [13-16].

Intuitionistic fuzzy sets (IFSs), as an extended form of fuzzy sets (FSs), not only consider the membership degree of an element to the set, but also consider the non-membership degree of an element, so it is widely used. Interval-valued intuitionistic fuzzy sets (IVIFSs) expanded the membership degree and non-membership degree of elements from the numerical value to the subset of the interval $[0,1]$. These extended fuzzy sets solved many practical problems and attracted more and more scholars to research them. As a good tool to solve incomplete, uncertain and inconsistent 
problems, neutrosophic sets (NSs) were first proposed by Smarandache $[17,18]$. In solving practical problems, neutrosophic sets not only consider the membership degree and non-membership degree of elements, but also consider the uncertainty and inconsistency of elements. Compared with fuzzy sets and intuitionistic fuzzy sets, neutrosophic sets express the incompleteness and inconsistency of an element to set, which is more suitable for solving practical problems. However, all functions of neutrosophic sets are defined on a non-standard interval $]^{-} 0,1^{+}[\text {, where } T(x), I(x), F(x): X \rightarrow]^{-} 0,1^{+}$, and $T(x), I(x)$ and $F(x)$ satisfy the inequality: ${ }^{-} 0 \leq T(x)+I(x)+F(x) \leq 3^{+}$. The non-standard interval of neutrosophic sets gives it some defects in solving many practical problems in real life. Therefore, many extended forms of neutrosophic sets (NSs) have been proposed successively, such as single value neutrosophic sets (SVNSs) [19] and interval neutrosophic sets (INSs) [20], and so forth, and have been applied to many practical problems [21-23]. In 2013, F. Smarandache [20] proposed single-valued neutrosophic sets(SVNSs). The single-valued neutrosophic sets defined the value of the function of the neutrosophic sets in the standard interval $[0,1]$. Therefore, single-valued neutrosophic sets have a good application. Huang [24] proposed the distance measure and similarity measure of single-valued neutrosophic sets, and illustrated its application in decision-making by numerical examples. In 2017, Shahzadi [25] proposed the application of distance measure and similarity measure of single-valued neutrosophic sets and applied them to medical diagnosis. In 2013, Ye [26] proposed the application of correlation coefficients of single-valued neutrosophic sets in multi-attribute decision making problems. In order to better describe the uncertainty, incompleteness and inconsistency of many problems in life, Wang et al. [20] proposed interval neutrosophic sets. Then, Broumi [27] introduced correlation coefficient of interval neutrosophic sets. Chi [28] put forward the application of interval neutrosophic sets in multi-attribute decision making.

In 2010, Young [29] proposed the definition of cubic sets (CSs) and discussed its related properties. By extending cubic sets to neutrosophic sets, Young [30] put forward the concept of neutrosophic cubic sets (NCSs), defined internal and external neutrosophic cubic sets and discussed its related properties. Subsequently, some researchers have further studied the neutrosophic cubic sets. Tu [31] proposed the application of a similarity measure of neutrosophic cubic sets in multi-attribute decision making. Ye [32] proposed the application of the aggregation operator of neutrosophic cubic sets in multi-attribute decision making. Ye [33] also put forward the R-union of the neutrosophic cubic sets and R-intersection of neutrosophic cubic sets. In 2019, Xue [34] proposed the correlation coefficient of neutrosophic cubic sets and illustrated its application in decision-making and pattern recognition by numerical examples.

In multiple attribute decision making problems, the contain probability of an element will produce significant influence the result of the decision. The neutrosophic cubic sets only studied the membership degree, non-membership degree and uncertainty of an element, but there is no research on the probability that an element belongs to a set, therefore, this paper puts forward the concept of possibility neutrosophic cubic sets, studies some related concepts of possibility neutrosophic cubic sets, and the concrete examples illustrate the rationality of them. Finally, according to the score function, as an important indicator in the multi-attribute decision-making problem, and combining the scoring function index, we give a solution to solve the multiple attribute decision making problems in possibility neutrosophic cubic sets, and the specific numerical example illustrates the effectiveness of the method. This paper considers the application of neutrosophic cubic sets in multi-attribute decision making problems from a new point of view, which makes the decision process more comprehensive and the decision result better.

Possibility neutrosophic cubic sets is a generalization of neutrosophic cubic sets. The neutrosophic cubic sets consider the uncertainty, inconsistencies and incompleteness of the problem when solving practical problems, so it has been widely studied by many researchers. On the basis of testing the uncertainty, inconsistencies and incompleteness, the possibility neutrosophic cubic sets is added according to the specific situation, which makes the solution of the problem more in line with the objective facts. In neutrosophic cubic sets, the possibility of each element is regarded as 1 , so that the 
weight of each element is equal. However, in the possibility neutrosophic cubic sets, the possibility of each element is considered to be in the interval of $[0,1]$, which conforms to the objective situation.

Alkhazaleh [35] introduced the definition and properties of possibility fuzzy soft set in 2015, and applied it to multi-attribute decision making problems. Bashir [36] proposed a possibility intuitionistic fuzzy soft set and studied its application in decision-making problems. Yang [37] proposed the definition of a possibility interval valued fuzzy soft set and discussed the related properties and operator operations, and illustrated the application of a possibility fuzzy soft set by numerical examples. In 2014, Karaaslan [38,39] proposed the definition of possibility neutrosophic soft fuzzy sets, discussed its operation and properties, and studied its application in multi-attribute decision-making. Hassan [40] introduced some related concepts of possibility neutrosophic soft set, and put forward the possibility neutrosophic soft decision method. These sets of introduced probabilities do prove to be more efficient at solving real problems.

In summary, the main contributions of this article are as follows:

First, in this paper, we put forward a new concept of possibility neutrosophic cubic sets, and the empty sets, complete sets, complement sets, distance measure and similarity measure of the possibility neutrosophic cubic sets are defined. At the same time, we give the binary operation of possibility neutrosophic cubic sets.

Second, possibility neutrosophic cubic sets not only consider the membership, non-membership and uncertainty of elements, but also consider the possibility of each element in the set, giving different weights to elements, making the distribution of elements closer to the overall level of the research problem, so as to make the decision result more real.

Third, a simple linear model is proposed to solve the multi-attribute decision-making problem, which makes the solution more convenient and relatively simple.

The structure of this article is as follows-in Section 2, the basic concepts of CSs, NSs, SVNSs, INSs and NCSs are given. The related concepts and properties of possibility neutrosophic cubic sets are proposed in Section 3. In Section 4, a decision method is given and the feasibility and superiority of the method is illustrated by comparative analysis. Section 5 concludes the paper.

\section{Preliminaries}

In this section, we review some definitions to prepare for this article. We first introduce some of the basic concepts, including fuzzy sets (FSs), interval fuzzy sets (IFSs), neutrosophic sets (NSs), single valued neutrosophic sets( SVNSs), interval neutrosophic sets (INSs), cubic sets (CSs) and neutrosophic cubic sets (NCSs), and so forth.

Definition 1. [1] Let $X$ be a universe, then the fuzzy sets (FSs) $\mu$ be defined as follows

$$
\mu=\{\mu(x) \mid x \in X\}
$$

where $\mu(x)$ is membership of $x$ to set $\mu$.

For two fuzzy sets $\mu(x), v(x)$ in $X$, the join $(\vee)$ and meet $(\wedge)$ of $\mu$ and $v$ are defined by

$$
\begin{aligned}
& (\mu \vee v)(x)=\max \{\mu(x), v(x)\}, \\
& (\mu \wedge v)(x)=\min \{\mu(x), v(x)\} .
\end{aligned}
$$

If the fuzzy sets $\mu(x)$ and $v(x)$ in $X$ satisfy inequality $\mu(x) \leq v(x)$, for all $x \in X$, we called $\mu(x)$ is the fuzzy subset of $v(x)$.

Definition 2. [10] Let X be an universe, then the interval value fuzzy sets (IVFs) $\Psi$ is defined as follows

$$
\Psi=\left\{\left[\Psi^{-}(x), \Psi^{+}(x)\right] \mid x \in X\right\},
$$


where $\left[\Psi^{-}(x), \Psi^{+}(x)\right]$ is membership of $x$ to set $\Psi$.

For any interval valued fuzzy sets $\Psi_{1}$, and $\Psi_{2}$ in $X$, the union and intersection of $\Psi_{1}$, and $\Psi_{2}$ are defined as follows:

$$
\begin{aligned}
\left(\Psi_{1} \cup \Psi_{2}\right)(x) & =\left[\max \left(\Psi_{1}^{-}(x), \Psi_{2}^{-}(x)\right), \max \left(\Psi_{1}^{+}(x), \Psi_{2}^{+}(x)\right)\right], \\
\left(\Psi_{1} \cap \Psi_{2}\right)(x) & =\left[\min \left(\Psi_{1}^{-}(x), \Psi_{2}^{-}(x)\right), \min \left(\Psi_{1}^{+}(x), \Psi_{2}^{+}(x)\right)\right] .
\end{aligned}
$$

Definition 3. [18] Let X be an universe. The form of neutrosophic sets (NSs) $\lambda$ in $X$ be defined as

$$
\lambda=\{<x, T(x), I(x), F(x)>\mid x \in X\},
$$

where $T, I, F: X \rightarrow]^{-} 0,1^{+}[$define the degree of truth membership function, indeterminacy membership function and falsehood membership function of $x \in X$ to $\lambda$, respectively, and ${ }^{-} 0 \leq T(x)+I(x)+F(x) \leq 3^{+}$.

Due to neutrosophic sets being defined on a non-standard interval, from a scientific or engineering perspective, the neutrosophic sets need to be specified. Otherwise, it will be difficult to apply in real applications.

Definition 4. [19] Let X be a universe. The form of single valued neutrosophic set (SVNS) $\Gamma$ in X be defined as

$$
\Gamma=\{<x, T(x), I(x), F(x)>\mid x \in X\},
$$

where $T, I, F: X \rightarrow[0,1]$ define the degree of truth membership function, indeterminacy membership function and falsehood membership function of $x \in X$ to $\Gamma$, respectively, and $0 \leq T(x)+I(x)+F(x) \leq 3$.

Definition 5. [20] Let X be a universe. An interval neutrosophic set (INS) A in X is a structure of the form:

$$
A=\left\{<x, A_{T}(x), A_{I}(x), A_{F}(x)>\mid x \in X\right\} .
$$

where $A_{T}, A_{I}$, and $A_{F}$ are interval-valued fuzzy sets in $X$, respectively, which are called an interval truth membership function, an interval indeterminacy membership function and an interval falsity membership function, respectively.

Definition 6. [29] let X be a universe, then a cubic set (CS) has the following form

$$
\Omega=\{<x, \Psi(x), \mu(x)>\mid x \in X\},
$$

in which $\Psi$ is an interval value fuzzy set (IVF) and $\mu$ is a fuzzy set (FS).

Definition 7. [30] Let X be a universe, the neutrosophic cubic sets (NCSs) $\Phi$ is defined as:

$$
\Phi=\{<x, A(x), \lambda(x)>\mid x \in X\},
$$

where $A$ is an INS and $\lambda$ is an NS. An NCS can be denoted as $\Phi=\langle A, \lambda\rangle$.

Definition 8. [30] Let $X$ be a universe, $\Phi_{A}=\{A, \lambda\}$ and $\Phi_{B}=\{B, \beta\}$ are two NCSs in $X$ where

$$
\begin{gathered}
\Phi_{A}=\{<x, A(x), \lambda(x)>\mid x \in X\}, \\
\Phi_{B}=\{<x, B(x), \beta(x)>\mid x \in X\} .
\end{gathered}
$$

then,

(a) $($ Equality $) \Phi_{A}=\Phi_{B} \Longleftrightarrow A=B$ and $\lambda=\beta$. 
(b) $(R$-order $) \Phi_{A} \subseteq_{R} \Phi_{B} \Longleftrightarrow A \subseteq B$ and $\lambda \geq \beta$.

Definition 9. [30] let $X$ be a universe, for neutrosophic cubic sets $\Phi_{i}=\left\{A_{i}, \lambda_{i}\right\}$ where

$$
\begin{gathered}
A_{i}=\left\{<A_{i T}(x), A_{i I}(x), A_{i F}(x)>\mid x \in X\right\}, \\
\lambda_{i}=\left\{<\lambda_{i T}(x), \lambda_{i I}(x), \lambda_{i F}(x)>\mid x \in X\right\},
\end{gathered}
$$

for $i \in J$ and $J$ is any index set, we define

where

(1) $\bigcup_{R} \Phi_{i}=\left(\bigcup A_{i}, \wedge \lambda_{i}\right) \quad(R-$ union $)$

(2) $\bigcap_{R} \Phi_{i}=\left(\cap A_{i}, \bigvee \lambda_{i}\right) \quad(R-$ intersection $)$

$$
\begin{aligned}
& \cup A_{i}=\left\{<\left(\bigcup A_{i T}\right)(x),\left(\bigcup A_{i I}\right)(x),\left(\bigcup A_{i F}\right)(x)>\mid x \in X\right\}, \\
& \bigvee \lambda_{i}=\left\{<\left(\bigvee \lambda_{i T}\right)(x),\left(\bigvee \lambda_{i I}\right)(x),\left(\bigvee \lambda_{i F}\right)(x)>\mid x \in X\right\}, \\
& \cap A_{i}=\left\{<\left(\cap A_{i T}\right)(x),\left(\cap A_{i I}\right)(x),\left(\cap A_{i F}\right)(x)>\mid x \in X\right\}, \\
& \wedge \lambda_{i}=\left\{<\left(\bigwedge \lambda_{i T}\right)(x),\left(\bigwedge \lambda_{i I}\right)(x),\left(\bigwedge \lambda_{i F}\right)(x)>\mid x \in X\right\} .
\end{aligned}
$$

Definition 10. [30] Let $X$ be a universe, $\Phi$ is a NCS. Then, the complement of $\Phi=\{A, \lambda\}$ is defined to be the neutrosophic cubic set $\Phi^{c}=\left\{A^{c}, \lambda^{c}\right\}$ where $A^{c}=\left\{<A_{T}^{c}(x), A_{I}^{c}(x), A_{F}^{c}(x)>\mid x \in X\right\}$ is an interval neutrosophic set in $X$ and $\lambda^{c}=\left\{<\lambda_{T}^{c}(x), \lambda_{I}^{c}(x), \lambda_{F}^{c}(x)>\mid x \in X\right\}$ is a neutrosophic set in $X$.

Definition 11. [30] Let $X$ be a universe, $\Phi_{A}=\{A, \lambda\}, \Phi_{B}=\{B, \beta\}, \Phi_{C}=\{C, \gamma\}$ and $\Phi_{D}=\{D, \delta\}$ are neutrosophic cubic sets, then we have

(1) if $\Phi_{A} \subseteq_{R} \Phi_{B}$ and $\Phi_{B} \subseteq_{R} \Phi_{C}$, then $\Phi_{A} \subseteq_{R} \Phi_{C}$.

(2) if $\Phi_{A} \subseteq_{R} \Phi_{B}$ then $\left(\Phi_{B}\right)^{c} \subseteq_{R}\left(\Phi_{A}\right)^{c}$.

(3) if $\Phi_{A} \subseteq_{R} \Phi_{B}$ and $\Phi_{A} \subseteq_{R} \Phi_{C}$, then $\Phi_{A} \subseteq_{R} \Phi_{B} \cap_{R} \Phi_{C}$.

(4) if $\Phi_{A} \subseteq_{R} \Phi_{B}$ and $\Phi_{C} \subseteq_{R} \Phi_{B}$, then $\Phi_{A} \cup_{R} \Phi_{C} \subseteq_{R} \Phi_{B}$.

(5) if $\Phi_{A} \subseteq_{R} \Phi_{B}$ and $\Phi_{C} \subseteq_{R} \Phi_{D}$, then $\Phi_{A} \cup_{R} \Phi_{C} \subseteq_{R} \Phi_{B} \cup_{R} \Phi_{D}$ and $\Phi_{A} \cap_{R} \Phi_{C} \subseteq_{R} \Phi_{B} \cap_{R} \Phi_{D}$.

\section{Possibility Neutrosophic Cubic Sets}

In this part, we propose the definition of possibility neutrosophic cubic sets, give some related definitions and binary operation of possibility neutrosophic cubic sets, and discuss its related properties.

Definition 12. Let $X$ be a universe, $E$ be an attribute set, then a possibility neutrosophic cubic sets $\mathscr{P}_{X}$ over $X$ has the form as follows

$$
\mathscr{P}_{X}=\left\{\left(x,<A_{T}(x), A_{I}(x), A_{F}(x)>,<T(x), I(x), F(x)>, \mu(x)\right) \mid x \in X\right\}
$$

where $A_{T}(x), A_{I}(x)$ and $A_{F}(x)$ is interval-valued fuzzy sets, $T(x), I(x)$ and $F(x)$ is fuzzy sets, $\mu(x)$ is the possibility of element $x \in X$ to $\mathscr{P}_{X}$, denoted by $\mathscr{P}_{X}=\left(\Phi_{A}, \mu\right)$.

Example 1. Let $X=\left\{x_{1}, x_{2}, x_{3}\right\}$ be a set of three companies, and let $E=\left\{e_{1}, e_{2}, e_{3}\right\}$ be a set of attributes, where $e_{1}=$ risk, $e_{2}=$ growth rate, and $e_{3}=$ environment. According to the above definition, when the decision-maker evaluates alternative $x_{1}$ under attribute $e_{1}$, the evaluation information given as follows:

$$
\mathscr{P}_{X}\left(x_{1}\right)\left(e_{1}\right)=\{\langle[0.6,0.8],[0.1,0.2],[0.2,0.3]\rangle,\langle 0.7,0.1,0.2\rangle, 0.7\} .
$$


The evaluation value for alternative under all attributes constitutes the possibility neutrosophic cubic sets are as follows

$$
\mathscr{P}_{X}\left(x_{1}\right)=\left[\begin{array}{l}
(\langle[0.6,0.8],[0.1,0.2],[0.2,0.3]\rangle,\langle 0.7,0.1,0.2\rangle, 0.7) \\
(\langle[0.6,0.7],[0.1,0.2],[0.2,0.3]\rangle,\langle 0.6,0.1,0.2\rangle, 0.6) \\
(\langle[0.6,0.7],[0.3,0.4],[0.1,0.2]\rangle,\langle 0.7,0.4,0.1\rangle, 0.8)
\end{array}\right] .
$$

Similarly, the evaluation value of different alternatives under the same attribute can also be given in the form of possibility neutrosophic cubic sets,

$$
\begin{gathered}
\mathscr{P}_{X}\left(x_{2}\right)=\left[\begin{array}{l}
(\langle[0.4,0.6],[0.2,0.3],[0.1,0.3]\rangle,\langle 0.6,0.2,0.2\rangle, 0.5) \\
(\langle[0.5,0.6],[0.2,0.3],[0.3,0.4]\rangle,\langle 0.6,0.3,0.4\rangle, 0.8) \\
(\langle[0.5,0.7],[0.2,0.3],[0.3,0.4]\rangle,\langle 0.6,0.2,0.3\rangle, 0.7)
\end{array}\right], \\
\mathscr{P}_{X}\left(x_{3}\right)=\left[\begin{array}{l}
(\langle[0.7,0.8],[0.1,0.2],[0.1,0.2]\rangle,\langle 0.8,0.1,0.2\rangle, 0.6) \\
(\langle[0.6,0.7],[0.1,0.2],[0.1,0.3]\rangle,\langle 0.7,0.1,0.2\rangle, 0.7) \\
(\langle[0.6,0.7],[0.3,0.4],[0.2,0.3]\rangle,\langle 0.7,0.3,0.2\rangle, 0.6)
\end{array}\right] .
\end{gathered}
$$

For the same alternative, different decision makers may give different possibility neutrosophic cubic sets, such as

$$
\begin{aligned}
\mathscr{Q}_{X}\left(x_{1}\right)=\left[\begin{array}{l}
(\langle[0.5,0.7],[0.1,0.3],[0.1,0.2]\rangle,\langle 0.6,0.1,0.3\rangle, 0.7) \\
(\langle[0.7,0.8],[0.1,0.2],[0.2,0.3]\rangle,\langle 0.8,0.1,0.1\rangle, 0.6) \\
(\langle[0.7,0.9],[0.2,0.3],[0.1,0.2]\rangle,\langle 0.7,0.2,0.3\rangle, 0.8)
\end{array}\right], \\
\mathscr{Q}_{X}\left(x_{2}\right)=\left[\begin{array}{l}
(\langle[0.5,0.6],[0.2,0.3],[0.1,0.3]\rangle,\langle 0.6,0.2,0.2\rangle, 0.5) \\
(\langle[0.6,0.7],[0.1,0.3],[0.2,0.4]\rangle,\langle 0.6,0.1,0.4\rangle, 0.8) \\
(\langle[0.5,0.7],[0.2,0.3],[0.1,0.3]\rangle,\langle 0.7,0.2,0.3\rangle, 0.8)
\end{array}\right], \\
\mathscr{Q}_{X}\left(x_{3}\right)=\left[\begin{array}{l}
(\langle[0.7,0.8],[0.1,0.2],[0.1,0.2]\rangle,\langle 0.8,0.1,0.2\rangle, 0.6) \\
(\langle[0.6,0.7],[0.1,0.2],[0.1,0.3]\rangle,\langle 0.7,0.1,0.2\rangle, 0.5) \\
(\langle[0.8,0.9],[0.2,0.4],[0.1,0.2]\rangle,\langle 0.9,0.2,0.2\rangle, 0.8)
\end{array}\right],
\end{aligned}
$$

Definition 13. Let $\mathscr{P}_{X}=\left\{\left(x,<A_{T}(x), A_{I}(x), A_{F}(x)>,<T_{A}(x), I_{A}(x), F_{A}(x)>, \mu(x)\right) \mid x \in X\right\}$, $\mathscr{Q}_{X}=\left\{\left(x,<B_{T}(x), B_{I}(x), B_{F}(x)>,<T_{B}(x), I_{B}(x), F_{B}(x)>, v(x)\right) \mid x \in X\right\}$ are two possibility neutrosophic cubic sets, then $\mathscr{P}_{X}$ is called possibility neutrosophic cubic subsets of $\mathscr{Q}_{X}$, expressed by mathematical symbols such as $\mathscr{P}_{X} \subseteq \mathscr{Q}_{X}$, if

(1) $\mu(x) \leq v(x)$, for all $x \in X$;

(2) $A_{T}(x) \leq B_{T}(x), A_{I}(x) \geq B_{I}(x), A_{F}(x) \geq B_{F}(x), T_{A}(x) \leq T_{B}(x), I_{A}(x) \geq I_{B}(x)$, and $F_{A}(x) \geq F_{B}(x)$, for all $x \in X$.

Example 2. Let $X=\left\{x_{1}, x_{2}, x_{3}\right\}$ be a set of three schools, and let $E=\left\{e_{1}, e_{2}, e_{3}\right\}$ be a set of attributes, where $e_{1}=$ cost, $e_{2}=$ atmosphere and $e_{3}=$ teaching quality. The two experts give the possibility neutrosophic cubic sets for evaluating the value of each alternative under each attribute.

$$
\begin{gathered}
\mathscr{P}_{X}\left(x_{1}\right)=\left[\begin{array}{l}
(\langle[0.6,0.8],[0.1,0.2],[0.2,0.3]\rangle,\langle 0.7,0.1,0.2\rangle, 0.7) \\
(\langle[0.6,0.7],[0.1,0.2],[0.2,0.3]\rangle,\langle 0.6,0.1,0.2\rangle, 0.6) \\
(\langle[0.6,0.7],[0.3,0.4],[0.1,0.2]\rangle,\langle 0.7,0.4,0.1\rangle, 0.8)
\end{array}\right], \\
\mathscr{P}_{X}\left(x_{2}\right)=\left[\begin{array}{l}
(\langle[0.4,0.6],[0.2,0.3],[0.1,0.3]\rangle,\langle 0.6,0.2,0.2\rangle, 0.5) \\
(\langle[0.5,0.6],[0.2,0.3],[0.3,0.4]\rangle,\langle 0.6,0.3,0.4\rangle, 0.8) \\
(\langle[0.5,0.7],[0.2,0.3],[0.3,0.4]\rangle,\langle 0.6,0.2,0.3\rangle, 0.7)
\end{array}\right],
\end{gathered}
$$




$$
\mathscr{P}_{X}\left(x_{3}\right)=\left[\begin{array}{l}
(\langle[0.7,0.8],[0.1,0.2],[0.1,0.2]\rangle,\langle 0.8,0.1,0.2\rangle, 0.6) \\
(\langle[0.6,0.7],[0.1,0.2],[0.1,0.3]\rangle,\langle 0.7,0.1,0.2\rangle, 0.7) \\
(\langle[0.6,0.7],[0.3,0.4],[0.2,0.3]\rangle,\langle 0.7,0.3,0.2\rangle, 0.6)
\end{array}\right],
$$

And another decision maker gives the following possibility neutrosophic cubic set.

$$
\begin{aligned}
& \mathscr{Q}_{X}\left(x_{1}\right)=\left[\begin{array}{l}
(\langle[0.8,0.9],[0.0,0.1],[0.1,0.2]\rangle,\langle 0.8,0.0,0.1\rangle, 0.8) \\
(\langle[0.7,0.8],[0.0,0.1],[0.0,0.1]\rangle,\langle 0.7,0.0,0.1\rangle, 0.8) \\
(\langle[0.7,0.9],[0.2,0.3],[0.0,0.1]\rangle,\langle 0.8,0.2,0.0\rangle, 0.9)
\end{array}\right], \\
& \mathscr{Q}_{X}\left(x_{2}\right)=\left[\begin{array}{l}
(\langle[0.6,0.8],[0.1,0.2],[0.0,0.1]\rangle,\langle 0.7,0.1,0.1\rangle, 0.6) \\
(\langle[0.6,0.7],[0.1,0.2],[0.1,0.2]\rangle,\langle 0.7,0.1,0.2\rangle, 0.9) \\
(\langle[0.6,0.8],[0.1,0.2],[0.1,0.3]\rangle,\langle 0.7,0.1,0.2\rangle, 0.8)
\end{array}\right], \\
& \mathscr{Q}_{X}\left(x_{3}\right)=\left[\begin{array}{l}
(\langle[0.8,0.9],[0.0,0.1],[0.0,0.1]\rangle,\langle 0.9,0.0,0.1\rangle, 0.7) \\
(\langle[0.7,0.8],[0.0,0.1],[0.0,0.2]\rangle,\langle 0.8,0.1,0.1\rangle, 0.8) \\
(\langle[0.7,0.8],[0.2,0.3],[0.1,0.2]\rangle,\langle 0.8,0.1,0.1\rangle, 0.7)
\end{array}\right] .
\end{aligned}
$$

Then, according to the above definition we can get $\mathscr{P}_{X}\left(x_{i}\right)$ is a possibility neutrosophic cubic subset of $\mathscr{Q}_{X}\left(x_{i}\right),(i=1,2,3)$ expressed as $\mathscr{P}_{X} \subseteq \mathscr{Q}_{X}$.

Definition 14. Let $\mathscr{P}_{X}=\left(\Phi_{A}, \mu\right)$ and $\mathscr{Q}_{X}=\left(\Phi_{B}, v\right)$ be two possibility neutrosophic cubic sets, if $\Phi_{A}=\Phi_{B}$ and $\mu=v$, then $\mathscr{P}_{X}=\left(\Phi_{A}, \mu\right)$ and $\mathscr{Q}_{X}=\left(\Phi_{B}, v\right)$ are called equal, denoted by $\mathscr{P}_{X}=\mathscr{Q}_{X}$.

Combined with the concept of internal and external neutrosophic cubic sets [30], this paper gives the definition of possibility internal and external neutrosophic cubic sets.

Definition 15. Let $X$ be a universe, $\mathscr{P}_{X}=\left\{\left(x,<\left[A_{T}^{-}(x), A_{T}^{+}(x)\right],\left[A_{I}^{-}(x), A_{I}^{+}(x)\right],\left[A_{F}^{-}(x), A_{F}^{+}(x)\right]>\right.\right.$ $\left.\left.,<T_{A}(x), I_{A}(x), F_{A}(x)>, \mu(x)\right) \mid x \in X\right\}$ be a possibility neutrosophic cubic set in $X, \mathscr{P}_{X}$ is said to be possibility internal neutrosophic cubic set, if for all $x \in X$, we have

$$
\begin{aligned}
& T_{A}(x) \in\left[A_{T}^{-}(x), A_{T}^{+}(x)\right], \\
& I_{A}(x) \in\left[A_{I}^{-}(x), A_{I}^{+}(x)\right], \\
& F_{A}(x) \in\left[A_{F}^{-}(x), A_{F}^{+}(x)\right] .
\end{aligned}
$$

Example 3. Let

$$
\mathscr{P}_{X}=\left[\begin{array}{l}
(\langle[0.7,0.9],[0.0,0.1],[0.0,0.1]\rangle,\langle 0.8,0.0,0.1\rangle, 0.7) \\
(\langle[0.6,0.7],[0.1,0.2],[0.1,0.2]\rangle,\langle 0.7,0.1,0.2\rangle, 0.9) \\
(\langle[0.6,0.8],[0.1,0.2],[0.1,0.3]\rangle,\langle 0.7,0.1,0.2\rangle, 0.8)
\end{array}\right],
$$

be a possibility neutrosophic cubic set, then it is said to be a possibility internal neutrosophic cubic set.

Definition 16. Let $\mathrm{X}$ be a universe, $\mathscr{P}_{X}=\left\{\left(x,<\left[A_{T}^{-}(x), A_{T}^{+}(x)\right],\left[A_{I}^{-}(x), A_{I}^{+}(x)\right],\left[A_{F}^{-}(x), A_{F}^{+}(x)\right]>\right.\right.$ $\left.\left.,<T_{A}(x), I_{A}(x), F_{A}(x)>, \mu(x)\right) \mid x \in X\right\}$ be a possibility neutrosophic cubic set in $X, \mathscr{P}_{X}$ is said to be possibility external neutrosophic cubic set, if for all $x \in X$, we have

$$
\begin{gathered}
T_{A}(x) \notin\left[A_{T}^{-}(x), A_{T}^{+}(x)\right], \\
I_{A}(x) \notin\left[A_{I}^{-}(x), A_{I}^{+}(x)\right], \\
F_{A}(x) \notin\left[A_{F}^{-}(x), A_{F}^{+}(x)\right] .
\end{gathered}
$$


Example 4. Let

$$
\mathscr{P}_{X}=\left[\begin{array}{l}
(\langle[0.8,0.9],[0.1,0.2],[0.0,0.1]\rangle,\langle 0.7,0.3,0.2\rangle, 0.7) \\
(\langle[0.6,0.7],[0.1,0.2],[0.1,0.2]\rangle,\langle 0.8,0.5,0.3\rangle, 0.9) \\
(\langle[0.6,0.8],[0.1,0.2],[0.1,0.3]\rangle,\langle 0.5,0.3,0.5\rangle, 0.8)
\end{array}\right],
$$

be a possibility neutrosophic cubic set, then it is said to be a possibility external neutrosophic cubic set.

Property 1. Let $X$ be an universe, $\mathscr{P}_{X}=\left\{\left(x,<\left[A_{T}^{-}(x), A_{T}^{+}(x)\right],\left[A_{I}^{-}(x), A_{I}^{+}(x)\right],\left[A_{F}^{-}(x), A_{F}^{+}(x)\right]>,<\right.\right.$ $\left.\left.T_{A}(x), I_{A}(x), F_{A}(x)>, \mu(x)\right) \mid x \in X\right\}$ and $\mathscr{Q}_{X}=\left\{\left(x,<\left[B_{T}^{-}(x), B_{T}^{+}(x)\right],\left[B_{I}^{-}(x), B_{I}^{+}(x)\right],\left[B_{F}^{-}(x), B_{F}^{+}(x)\right]\right.\right.$ $\left.\left.>,<T_{B}(x), I_{B}(x), F_{B}(x)>, v(x)\right) \mid x \in X\right\}$ are two possibility internal neutrosophic cubic sets, if $\mathscr{P}_{X}$ and $\mathscr{Q}_{X}$ satisfy the following conditions for all $x \in X$

$$
\begin{aligned}
& \max \left\{A_{T}^{-}(x), B_{T}^{-}(x)\right\} \leq(\lambda \wedge \beta)(x), \\
& \max \left\{A_{I}^{-}(x), B_{I}^{-}(x)\right\} \leq(\lambda \wedge \beta)(x), \\
& \max \left\{A_{F}^{-}(x), B_{F}^{-}(x)\right\} \leq(\lambda \wedge \beta)(x) .
\end{aligned}
$$

Then, the R-union of $\mathscr{P}_{X}$ and $\mathscr{Q}_{X}$ still be possibility internal neutrosophic cubic sets, where

$$
\mathscr{P}_{X} \cup_{R} \mathscr{Q}_{X}=\left(\Phi_{A} \cap \Phi_{B}, \mu \wedge v\right) .
$$

in which $\lambda=<T_{A}(x), I_{A}(x), F_{A}(x)>, \beta=<T_{B}(x), I_{B}(x), F_{B}(x)>$.

Proof. Due to the $\mathscr{P}_{X}$ and $\mathscr{Q}_{X}$ are possibility internal neutrosophic cubic sets, then we have $T_{A}(x) \in$ $\left[A_{T}^{-}(x), A_{T}^{+}(x)\right], I_{A}(x) \in\left[A_{I}^{-}(x), A_{I}^{+}(x)\right], F_{A}(x) \in\left[A_{F}^{-}(x), A_{F}^{+}(x)\right]$, and $T_{B}(x) \in\left[B_{T}^{-}(x), B_{T}^{+}(x)\right]$, $I_{B}(x) \in\left[B_{I}^{-}(x), B_{I}^{+}(x)\right], F_{B}(x) \in\left[B_{F}^{-}(x), B_{F}^{+}(x)\right]$. According to the meet of fuzzy sets, we have $(\lambda \wedge$ $\beta)(x)=\left\{\min \left\{T_{A}, T_{B}\right\}, \min \left\{I_{A}, I_{B}\right\}, \min \left\{F_{A}, F_{B}\right\}\right\}$. By definition of possibility internal neutrosophic cubic sets, $\min \left\{T_{A}, T_{B}\right\} \leq \max \left\{T_{A}^{+}, T_{B}^{+}\right\}, \min \left\{I_{A}, I_{B}\right\} \leq \max \left\{I_{A}^{+}, I_{B}^{+}\right\}, \min \left\{F_{A}, F_{B}\right\} \leq \max \left\{F_{A}^{+}, F_{B}^{+}\right\}$. Combined with known conditions $\max \left\{A_{T}^{-}(x), B_{T}^{-}(x)\right\} \leq \min \left\{T_{A}, T_{B}\right\}, \max \left\{A_{I}^{-}(x), B_{I}^{-}(x)\right\} \leq$ $\min \left\{T_{A}, T_{B}\right\}, \max \left\{A_{F}^{-}(x), B_{F}^{-}(x)\right\} \leq \min \left\{T_{A}, T_{B}\right\}$. Then we have the R-union of $\mathscr{P}_{X}$ and $\mathscr{Q}_{X}$ still be possibility internal neutrosophic cubic sets.

Example 5. Let

$$
\mathscr{P}_{X}=\left[\begin{array}{l}
(\langle[0.8,0.9],[0.1,0.2],[0.0,0.1]\rangle,\langle 0.8,0.2,0.1\rangle, 0.7) \\
(\langle[0.6,0.7],[0.1,0.2],[0.1,0.2]\rangle,\langle 0.6,0.2,0.1\rangle, 0.8) \\
(\langle[0.6,0.8],[0.1,0.2],[0.1,0.3]\rangle,\langle 0.8,0.2,0.2\rangle, 0.8)
\end{array}\right]
$$

and

$$
\mathscr{Q}_{X}=\left[\begin{array}{l}
(\langle[0.6,0.7],[0.1,0.2],[0.2,0.3]\rangle,\langle 0.7,0.2,0.3\rangle, 0.7) \\
(\langle[0.7,0.9],[0.3,0.5],[0.1,0.2]\rangle,\langle 0.8,0.5,0.2\rangle, 0.9) \\
(\langle[0.6,0.8],[0.2,0.3],[0.1,0.3]\rangle,\langle 0.7,0.3,0.2\rangle, 0.6)
\end{array}\right],
$$

be two possibility internal neutrosophic cubic sets, and satisfy the conditions in the above definition. Then

$$
\mathscr{P}_{X} \cup_{R} \mathscr{Q}_{X}=\left[\begin{array}{l}
(\langle[0.6,0.7],[0.1,0.2],[0.0,0.1]\rangle,\langle 0.7,0.2,0.1\rangle, 0.7) \\
(\langle[0.6,0.7],[0.1,0.2],[0.1,0.2]\rangle,\langle 0.6,0.2,0.1\rangle, 0.8) \\
(\langle[0.6,0.8],[0.1,0.2],[0.1,0.3]\rangle,\langle 0.7,0.2,0.2\rangle, 0.6)
\end{array}\right],
$$

be possibility internal neutrosophic cubic set.

Property 2. Let $X$ be an universe, $\mathscr{P}_{X}=\left\{\left(x,<\left[A_{T}^{-}(x), A_{T}^{+}(x)\right],\left[A_{I}^{-}(x), A_{I}^{+}(x)\right],\left[A_{F}^{-}(x), A_{F}^{+}(x)\right]>,<\right.\right.$ $\left.\left.T_{A}(x), I_{A}(x), F_{A}(x)>, \mu(x)\right) \mid x \in X\right\}$ and $\mathscr{Q}_{X}=\left\{\left(x,<\left[B_{T}^{-}(x), B_{T}^{+}(x)\right],\left[B_{I}^{-}(x), B_{I}^{+}(x)\right],\left[B_{F}^{-}(x), B_{F}^{+}(x)\right]\right.\right.$ 
$\left.\left.>,<T_{B}(x), I_{B}(x), F_{B}(x)>, v(x)\right) \mid x \in X\right\}$ are two possibility internal neutrosophic cubic sets, if $\mathscr{P}_{X}$ and $\mathscr{Q}_{X}$ satisfy the following condition for all $x \in X$

$$
\begin{aligned}
& (\lambda \vee \beta)(x) \leq \min \left\{A_{T}^{+}(x), B_{T}^{+}(x)\right\}, \\
& (\lambda \vee \beta)(x) \leq \min \left\{A_{I}^{+}(x), B_{I}^{+}(x)\right\}, \\
& (\lambda \vee \beta)(x) \leq \max \left\{A_{F}^{+}(x), B_{F}^{+}(x)\right\} .
\end{aligned}
$$

Then, the R-intersection of $\mathscr{P}_{\mathrm{X}}$ and $\mathscr{Q}_{\mathrm{X}}$ are still possibility internal neutrosophic cubic set, where

$$
\mathscr{P}_{X} \cap_{R} \mathscr{Q}_{X}=\left(\Phi_{A} \cap \Phi_{B}, \mu \vee v\right) .
$$

in which $\lambda=<T_{A}(x), I_{A}(x), F_{A}(x)>, \beta=<T_{B}(x), I_{B}(x), F_{B}(x)>$.

Proof. Due to the $\mathscr{P}_{X}$ and $\mathscr{Q}_{X}$ being possibility internal neutrosophic cubic sets, then we have $T_{A}(x) \in\left[A_{T}^{-}(x), A_{T}^{+}(x)\right], I_{A}(x) \in\left[A_{I}^{-}(x), A_{I}^{+}(x)\right], F_{A}(x) \in\left[A_{F}^{-}(x), A_{F}^{+}(x)\right]$, and $T_{B}(x) \in\left[B_{T}^{-}(x), B_{T}^{+}(x)\right], I_{B}(x) \in\left[B_{I}^{-}(x), B_{I}^{+}(x)\right], F_{B}(x) \in\left[B_{F}^{-}(x), B_{F}^{+}(x)\right]$. According to the join of fuzzy sets, we have $(\lambda \vee \beta)(x)=\left\{\max \left\{T_{A}, T_{B}\right\}, \max \left\{I_{A}, I_{B}\right\}, \max \left\{F_{A}, F_{B}\right\}\right\}$. By the definition of possibility internal neutrosophic cubic sets, $\min \left\{A_{T}^{-}(x), B_{T}^{-}(x)\right\} \leq \max \left\{T_{A}(x), T_{B}(x)\right\}$, $\min \left\{A_{I}^{-}(x), B_{I}^{-}(x)\right\} \leq \max \left\{I_{A}(x), I_{B}(x)\right\}, \min \left\{A_{F}^{-}(x), B_{F}^{-}(x)\right\} \leq \max \left\{F_{A}(x), F_{B}(x)\right\}$. Combined with known conditions $\max \left\{T_{A}, T_{B}\right\} \leq \min \left\{A_{T}^{+}(x), B_{T}^{+}(x)\right\}, \max \left\{I_{A}, I_{B}\right\} \leq \min \left\{A_{I}^{+}(x), B_{I}^{+}(x)\right\}$, $\max \left\{F_{A}, F_{B}\right\} \leq \min \left\{A_{F}^{+}(x), B_{F}^{+}(x)\right\}$. Then we have the R-intersection of $\mathscr{P}_{X}$ and $\mathscr{Q}_{X}$ is still possibility internal neutrosophic cubic sets.

Example 6. Let

$$
\mathscr{P}_{X}=\left[\begin{array}{l}
(\langle[0.6,0.9],[0.2,0.3],[0.1,0.2]\rangle,\langle 0.8,0.2,0.1\rangle, 0.7) \\
(\langle[0.6,0.8],[0.1,0.2],[0.1,0.2]\rangle,\langle 0.6,0.2,0.1\rangle, 0.8) \\
(\langle[0.6,0.7],[0.1,0.2],[0.1,0.3]\rangle,\langle 0.6,0.2,0.2\rangle, 0.7)
\end{array}\right],
$$

and

$$
\mathscr{Q}_{X}=\left[\begin{array}{l}
(\langle[0.7,0.9],[0.3,0.4],[0.2,0.3]\rangle,\langle 0.7,0.3,0.3\rangle, 0.7) \\
(\langle[0.8,0.9],[0.2,0.3],[0.1,0.2]\rangle,\langle 0.8,0.2,0.2\rangle, 0.9) \\
(\langle[0.7,0.8],[0.1,0.3],[0.2,0.3]\rangle,\langle 0.7,0.3,0.2\rangle, 0.6)
\end{array}\right],
$$

be two possibility internal neutrosophic cubic sets, and satisfy the conditions in the above definition, then

$$
\mathscr{P}_{X} \cap_{R} \mathscr{Q}_{X}=\left[\begin{array}{l}
(\langle[0.6,0.9],[0.2,0.3],[0.1,0.2]\rangle,\langle 0.7,0.2,0.1\rangle, 0.7) \\
(\langle[0.6,0.8],[0.1,0.2],[0.1,0.2]\rangle,\langle 0.6,0.2,0.1\rangle, 0.9) \\
(\langle[0.6,0.7],[0.1,0.2],[0.1,0.3]\rangle,\langle 0.6,0.2,0.2\rangle, 0.7)
\end{array}\right],
$$

be possibility internal neutrosophic cubic set.

Definition 17. Let $\mathscr{P}_{X}$ be possibility neutrosophic cubic sets, then $\mathscr{P}_{X}$ is called possibility neutrosophic cubic empty sets, denoted by $\varnothing$, if $\forall e \in E, \varnothing=\{<[0,0],[1,1],[1,1]>,<0,1,1>, 0\}$.

Definition 18. Let $\mathscr{P}_{X}$ be possibility neutrosophic cubic set, then $\mathscr{P}_{X}$ be called possibility neutrosophic cubic complete sets, denoted by $U$, if $\forall e \in E, U=\{<[1,1],[0,0],[0,0]\rangle,\langle 1,0,0\rangle, 1\}$.

Definition 19. Let $\mathscr{P}_{X}=\left\{\left(x,<A_{T}(x), A_{I}(x), A_{F}(x)>,<T(x), I(x), F(x)>, \mu(x)\right) \mid x \in X\right\}$ be possibility neutrosophic cubic sets, then the complement of $\mathscr{P}_{X}$ denoted by $\mathscr{P}_{X}^{c}$, expressed as $\mathscr{P}_{X}^{c}=\{(x,<$ $\left.\left.A_{F}(x), 1-A_{I}(x), A_{T}(x)>,<F(x), 1-I(x), T(x)>, 1-\mu(x)\right) \mid x \in X\right\}$.

Proposition 1. Let $\mathscr{P}_{X}, \mathscr{Q}_{X}$, and $\mathscr{R}_{X}$ be possibility neutrosophic cubic sets. Then 
(1) $\varnothing \subseteq \mathscr{P}_{X} ;$

(2) $\mathscr{P}_{X} \subseteq U$;

(3) $\mathscr{P}_{X} \subseteq \mathscr{Q}_{X}$, and $\mathscr{Q}_{X} \subseteq \mathscr{R}_{X}$, then $\mathscr{P}_{X} \subseteq \mathscr{R}_{X}$;

(4) $\varnothing^{c}=U$;

(5) $U^{c}=\varnothing ;$

(6) $\left(\mathscr{P}_{X}^{c}\right)^{c}=\mathscr{P}_{X}$.

Property 3. Let $X$ be an universe, $\mathscr{P}_{X}=\left\{\Phi_{A}, \mu\right\}, \mathscr{Q}_{X}=\left\{\Phi_{B}, v\right\}, \mathscr{W}_{X}=\left\{\Phi_{C}, \gamma\right\}$ and $\mathscr{R}_{X}=\left\{\Phi_{D}, \delta\right\}$ are possibility neutrosophic cubic sets in $X$, then we have

(1) if $\mathscr{P}_{X} \subseteq_{R} \mathscr{Q}_{X}$ and $\mathscr{Q}_{X} \subseteq_{R} \mathscr{W}_{X}$, then $\mathscr{P}_{X} \subseteq_{R} \mathscr{W}_{X}$;

(2) if $\mathscr{P}_{X} \subseteq_{R} \mathscr{Q}_{X}$, then $\left(\mathscr{Q}_{X}\right)^{c} \subseteq_{R}\left(\mathscr{P}_{X}\right)^{c}$;

(3) if $\mathscr{P}_{X} \subseteq_{R} \mathscr{Q}_{X}$ and $\mathscr{P}_{X} \subseteq_{R} \mathscr{W}_{X}$, then $\mathscr{P}_{X} \subseteq_{R} \mathscr{Q}_{X} \cap_{R} \mathscr{W}_{X}$;

(4) if $\mathscr{P}_{X} \subseteq_{R} \mathscr{Q}_{X}$ and $\mathscr{W}_{X} \subseteq_{R} \mathscr{Q}_{X}$, then $\mathscr{P}_{X} \cup_{R} \mathscr{W}_{X} \subseteq_{R} \mathscr{Q}_{X}$;

(5)if $\mathscr{P}_{X} \subseteq_{R} \mathscr{Q}_{X}$ and $\mathscr{W}_{X} \subseteq_{R} \mathscr{R}_{X}$, then $\mathscr{P}_{X} \cup_{R} \mathscr{W}_{X} \subseteq_{R} \mathscr{Q}_{X} \cup_{R} \mathscr{R}_{X}$ and $\mathscr{P}_{X} \cap_{R} \mathscr{W}_{X} \subseteq_{R} \mathscr{Q}_{X} \cap_{R} \mathscr{R}_{X}$.

Proof. (1) For $\mathscr{P}_{X}=\left\{\Phi_{A}, \mu\right\}$, and $\mathscr{Q}_{X}=\left\{\Phi_{B}, \nu\right\}$, due to $\Phi_{A}$ and $\Phi_{B}$ are two neutrosophic cubic sets, according to the definition of R-order of neutrosophic cubic sets, if $\mathscr{P}_{X} \subseteq_{R} \mathscr{Q}_{X}$ and $\mathscr{Q}_{X} \subseteq_{R} \mathscr{W}_{X}$, then we have $\Phi_{A} \subseteq_{R} \Phi_{B}, \mu \leq v$, and $\Phi_{B} \subseteq_{R} \Phi_{C}, v \leq \gamma$. Therefore $\Phi_{A} \subseteq_{R} \Phi_{B} \subseteq_{R} \Phi_{C}, \mu \leq v \leq \gamma$, that is to say $\Phi_{A} \subseteq_{R} \Phi_{C}, \mu \leq \gamma$. Hence, we have $\mathscr{P}_{X} \subseteq_{R} \mathscr{W}_{X}$.

(2) Because $\mathscr{P}_{X} \subseteq_{R} \mathscr{Q}_{X}$, According to the definition of possibility neutrosophic cubic sets complement, it is easy to prove $\left(\mathscr{Q}_{X}\right)^{c} \subseteq_{R}\left(\mathscr{P}_{X}\right)^{c}$.

(3) if $\mathscr{P}_{X} \subseteq_{R} \mathscr{Q}_{X}$ and $\mathscr{P}_{X} \subseteq_{R} \mathscr{W}_{X}$, there is $\Phi_{A} \subseteq_{R} \Phi_{B}, \mu \leq v$, and $\Phi_{A} \subseteq_{R} \Phi_{C}, v \leq \gamma$. According to the meet of neutrosophic cubic sets and fuzzy sets, we can get $\Phi_{A} \subseteq_{R} \Phi_{B} \cap \Phi_{C}, \mu \leq v \wedge \gamma$. And that proves $\mathscr{P}_{X} \subseteq_{R} \mathscr{Q}_{X} \cap_{R} \mathscr{W}_{X}$.

(4) if $\mathscr{P}_{X} \subseteq_{R} \mathscr{Q}_{X}$ and $\mathscr{W}_{X} \subseteq_{R} \mathscr{Q}_{X}$, then easy to get $\Phi_{A} \subseteq_{R} \Phi_{B}, \mu \leq v$, and $\Phi_{C} \subseteq_{R} \Phi_{B}, \gamma \leq v$. According to the join of neutrosophic cubic sets and fuzzy sets, we have $\Phi_{A} \cup \Phi_{C} \subseteq_{R} \Phi_{B}, \mu \vee \gamma \leq v$. So we can get the result $\mathscr{P}_{\mathrm{X}} \cup_{R} \mathscr{W}_{\mathrm{X}} \subseteq_{R} \mathscr{Q}_{\mathrm{X}}$.

(5) By combining the proofs of (3) and (4), we can prove that (5) of the Property is true.

In 2017, Ye [41] defined the concept of linguistic neutrosophic cubic sets and proposed the multi-attribute decision-making method of linguistic neutrosophic cubic sets. According to the content of Ye [41], this paper gives the score function of the possibility neutrosophic cubic sets, and the accuracy function and the certainty function as follows.

Definition 20. Let $X=\left\{x_{1}, x_{2}, \cdots, x_{n}\right\}$, and $\mathscr{P}_{X}=\left\{\left(x,<\left[A_{T}^{-}(x), A_{T}^{+}(x)\right],\left[A_{I}^{-}(x), A_{I}^{+}(x)\right],\left[A_{F}^{-}(x)\right.\right.\right.$, $\left.\left.A_{F}^{+}(x)\right]>,\langle T(x), I(x), F(x)>, \mu(x)) \mid x \in X\right\}$ be possibility neutrosophic cubic sets, then its score function, accuracy function and certainty function of $\mathscr{P}_{X}$ be defined as follows:

$$
\begin{gathered}
S\left(\mathscr{P}_{X}\right)=\sum_{i=1}^{n}\left[\left(4+A_{T}^{-}\left(x_{i}\right)+A_{T}^{+}\left(x_{i}\right)-A_{I}^{-}\left(x_{i}\right)-A_{I}^{+}\left(x_{i}\right)-A_{F}^{-}\left(x_{i}\right)-A_{F}^{+}\left(x_{i}\right)\right)+\left(2+T\left(x_{i}\right)-I\left(x_{i}\right)-F\left(x_{i}\right)\right)+\mu\left(x_{i}\right)\right] / 10 \\
A\left(\mathscr{P}_{X}\right)=\sum_{i=1}^{n}\left[A_{T}^{-}\left(x_{i}\right)+A_{T}^{+}\left(x_{i}\right)-A_{F}^{-}\left(x_{i}\right)-A_{F}^{+}\left(x_{i}\right)+\left(T\left(x_{i}\right)-F\left(x_{i}\right)\right)+\mu\left(x_{i}\right)\right] / 4 \\
C\left(\mathscr{P}_{X}\right)=\sum_{i=1}^{n}\left[A_{T}^{-}\left(x_{i}\right)+A_{T}^{+}\left(x_{i}\right)+T\left(x_{i}\right)+\mu\left(x_{i}\right)\right] / 4
\end{gathered}
$$

The score function and accuracy function are two important indexes, which can be used to judge the size of the relationship between two possibility neutrosophic cubic sets. Generally, the higher the score, the greater the true membership of the possibility neutrosophic cubic sets. If the score functions of two possibility neutrosophic cubic sets are equal, we can judge their size relationship by comparing their accuracy function. If the accuracy function is large, the possibility neutrosophic cubic set will 
be better. For the certainty function, the larger the true membership function and possibility value, the more certainty the possibility neutrosophic cubic sets have.

Based on the above definition, the comparison and ranking of possibility neutrosophic cubic sets are defined as follows:

Definition 21. Let $X=\left\{x_{1}, x_{2}, \cdots, x_{n}\right\}, \mathscr{P}_{X}=\left\{\left(x,<\left[A_{T}^{-}(x), A_{T}^{+}(x)\right],\left[A_{I}^{-}(x), A_{I}^{+}(x)\right],\left[A_{F}^{-}(x)\right.\right.\right.$, $\left.\left.\left.A_{F}^{+}(x)\right]>,<T_{A}(x), I_{A}(x), F_{A}(x)>, \mu(x)\right) \mid x \in X\right\}$ and $\mathscr{Q}_{X}=\left\{\left(x,<\left[B_{T}^{-}(x), B_{T}^{+}(x)\right],\left[B_{I}^{-}(x), B_{I}^{+}(x)\right]\right.\right.$, $\left.\left.\left[B_{F}^{-}(x), B_{F}^{+}(x)\right]>,<T_{B}(x), I_{B}(x), F_{B}(x)>, v(x)\right) \mid x \in X\right\}$ be two possibility neutrosophic cubic sets, then the ranking of $\mathscr{P}_{X}$ and $\mathscr{Q}_{X}$ are defined as follows:

(1) If $S\left(\mathscr{P}_{X}\right)>S\left(\mathscr{Q}_{\mathrm{X}}\right)$, then $\mathscr{P}_{\mathrm{X}}>\mathscr{Q}_{\mathrm{X}}$;

(2) If $S\left(\mathscr{P}_{\mathrm{X}}\right)=S\left(\mathscr{Q}_{\mathrm{X}}\right)$ and $A\left(\mathscr{P}_{\mathrm{X}}\right)>A\left(\mathscr{Q}_{\mathrm{X}}\right)$, then $\mathscr{P}_{\mathrm{X}}>\mathscr{Q}_{\mathrm{X}}$.

Example 7. Let $X=\{x\}$ be an universe, $\mathscr{P}_{X}$ and $\mathscr{Q}_{X}$ are two possibility neutrosophic cubic sets.

(1) Suppose $\mathscr{P}_{X}=(\langle[0.8,0.9],[0.0,0.1],[0.0,0.1]\rangle,\langle 0.9,0.0,0.1\rangle, 0.7)$ and $\mathscr{Q}_{X}=$ $(\langle[0.7,0.8],[0.0,0.1],[0.0,0.2]\rangle,\langle 0.8,0.1,0.1\rangle, 0.8)$. From the definition we can get $S\left(\mathscr{P}_{X}\right)=0.9, S\left(\mathscr{Q}_{X}\right)=$ 0.86 , then $S\left(\mathscr{P}_{X}\right)>S\left(\mathscr{Q}_{X}\right)$, therefore $\mathscr{P}_{X}>\mathscr{Q}_{X}$.

(2) Suppose $\mathscr{P}_{X}=(\langle[0.5,0.8],[0.1,0.2],[0.0,0.1]\rangle,\langle 0.7,0.1,0.1\rangle, 0.6)$ and $\mathscr{Q}_{X}=$ $(\langle[0.6,0.7],[0.1,0.3],[0.1,0.2]\rangle,\langle 0.8,0.1,0.2\rangle, 0.9)$. From the definition we know $S\left(\mathscr{P}_{\mathrm{X}}\right)=0.8, S\left(\mathscr{Q}_{\mathrm{X}}\right)=$ $0.8, A\left(\mathscr{P}_{X}\right)=0.6, A\left(\mathscr{Q}_{X}\right)=0.625$, then $S\left(\mathscr{P}_{X}\right)=S\left(\mathscr{Q}_{X}\right), A\left(\mathscr{P}_{X}\right)<A\left(\mathscr{Q}_{X}\right)$, therefore $\mathscr{P}_{X}<\mathscr{Q}_{X}$.

(3) Suppose $\mathscr{P}_{\mathrm{X}}=(\langle[0.5,0.8],[0.1,0.2],[0.0,0.1]\rangle,\langle 0.7,0.1,0.1\rangle, 0.6)$ and $\mathscr{Q}_{\mathrm{X}}=$ $(\langle[0.6,0.7],[0.1,0.2],[0.1,0.2]\rangle,\langle 0.7,0.1,0.2\rangle, 0.9)$. From the definition we find $S\left(\mathscr{P}_{X}\right)=0.8, S\left(\mathscr{Q}_{X}\right)=$ $0.8, A\left(\mathscr{P}_{X}\right)=0.6, A\left(\mathscr{Q}_{X}\right)=0.6, C\left(\mathscr{P}_{X}\right)=0.65, C\left(\mathscr{Q}_{X}\right)=0.725$, then $S\left(\mathscr{P}_{X}\right)=S\left(\mathscr{Q}_{X}\right), A\left(\mathscr{P}_{X}\right)=$ $A\left(\mathscr{Q}_{\mathrm{X}}\right), C\left(\mathscr{P}_{\mathrm{X}}\right)<\mathrm{C}\left(\mathscr{Q}_{\mathrm{X}}\right)=0.725$, therefore $\mathscr{P}_{\mathrm{X}}<\mathscr{Q}_{\mathrm{X}}$.

Definition 22. [42] Let $g$ and $h$ be two real numbers, then the Dombi T-norm and T-conorm between $g$ and $h$ are defined as follows:

$$
\begin{gathered}
D(g, h)=\frac{1}{1+\left\{\left(\frac{1-g}{g}\right)^{\rho}+\left(\frac{1-h}{h}\right)^{\rho}\right\}^{\frac{1}{\rho}}}, \\
D^{c}(g, h)=1-\frac{1}{1+\left\{\left(\frac{1-g}{g}\right)^{\rho}+\left(\frac{1-h}{h}\right)^{\rho}\right\}^{\frac{1}{\rho}},}
\end{gathered}
$$

where $(g, h) \in(0,1) \times(0,1)$ and if $\rho>0$ then the operator $D(g, h)$ is conjunctive and $D^{c}(g, h)$ is disjunctive, satisfying $D(0,0)=D(0,1)=D(1,0)=0, D(1,1)=1, D^{c}(0,1)=D^{c}(1,0)=D^{c}(1,1)=1$ and $D^{c}(0,0)=0$ and if $\rho<0$ then the operator $D(g, h)$ is disjunctive and the operator $D^{c}(g, h)$ is conjunctive.

According to the above definition and operations of neutrosophic cubic numbers proposed by Ye [43] , the binary operation of two possibility neutrosophic cubic numbers are given as follows:

Definition 23. Let $X=\left\{x_{1}, x_{2}, \cdots, x_{n}\right\}$, be an universe, $\mathscr{P}_{X}=\left\{\left(x,<\left[A_{T}^{-}(x), A_{T}^{+}(x)\right],\left[A_{I}^{-}(x), A_{I}^{+}(x)\right]\right.\right.$, $\left.\left.\left[A_{F}^{-}(x), A_{F}^{+}(x)\right]>,<T_{A}(x), I_{A}(x), F_{A}(x)>, \mu(x)\right) \mid x \in X\right\}$ and $\mathscr{Q}_{X}=\left\{\left(x,<\left[B_{T}^{-}(x), B_{T}^{+}(x)\right]\right.\right.$, $\left.\left.\left[B_{I}^{-}(x), B_{I}^{+}(x)\right],\left[B_{F}^{-}(x), B_{F}^{+}(x)\right]>,<T_{B}(x), I_{B}(x), F_{B}(x)>, v(x)\right) \mid x \in X\right\}$ be two possibility neutrosophic cubic sets in $X, \rho>0$. Then the binary operations of two possibility neutrosophic cubic sets are given as follows:

(1) $\mathscr{P}_{X} \oplus \mathscr{Q}_{X}=$

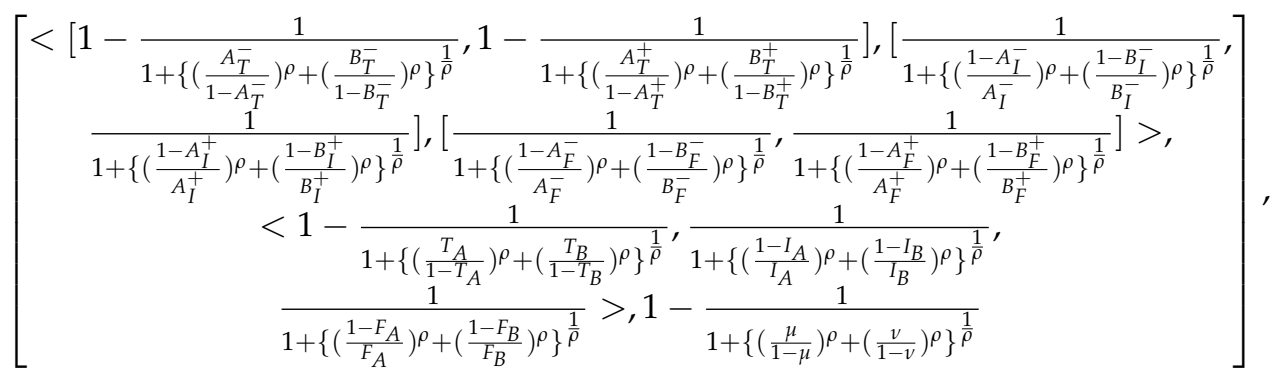




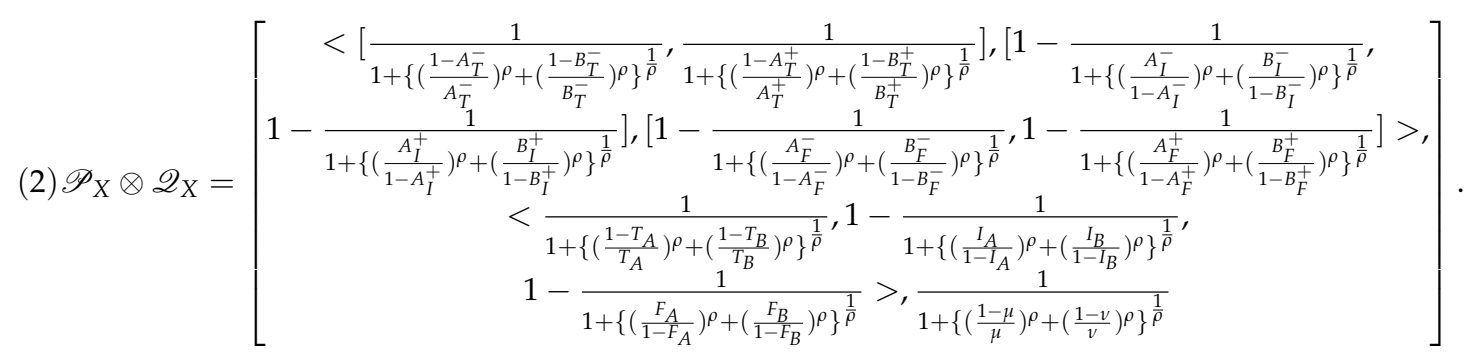

Example 8. Let $X=\{x\}$ be a universe, $\mathscr{P}_{X}, \mathscr{Q}_{X}$ are two possibility neutrosophic cubic sets. Suppose that $\mathscr{P}_{X}=(\langle[0.5,0.8],[0.1,0.2],[0.0,0.1]\rangle,\langle 0.7,0.1,0.1\rangle, 0.6), \mathscr{Q}_{X}=(\langle[0.6,0.7],[0.1,0.3],[0.1,0.2]\rangle$, $\langle 0.8,0.1,0.2\rangle, 0.9)$ and $\rho=1$. From the above definition we can get the following results.

$$
\begin{aligned}
& \mathscr{P}_{X} \oplus \mathscr{Q}_{X}=\{([0.71,0.86],[0.05,0.14],[0,0.07],<0.86,0.05,0.07>, 0.91)\} \\
& \mathscr{P}_{X} \otimes \mathscr{Q}_{X}=\{([0.38,0.60],[0.18,0.40],[0,0.80],<0.60,0.18,0.27>, 0.56)\}
\end{aligned}
$$

Definition 24. Let $X=\left\{x_{1}, x_{2}, \cdots, x_{n}\right\}$ be an universe, $\mathscr{P}_{X}=\left\{\left(x,<\left[A_{T}^{-}(x), A_{T}^{+}(x)\right],\left[A_{I}^{-}(x), A_{I}^{+}(x)\right]\right.\right.$, $\left.\left.\left[A_{F}^{-}(x), A_{F}^{+}(x)\right]>,<T_{A}(x), I_{A}(x), F_{A}(x)>, \mu(x)\right) \mid x \in X\right\}$ and $\mathscr{Q}_{X}=\left\{\left(x,<\left[B_{T}^{-}(x), B_{T}^{+}(x)\right]\right.\right.$, $\left.\left.\left[B_{I}^{-}(x), B_{I}^{+}(x)\right],\left[B_{F}^{-}(x), B_{F}^{+}(x)\right]>,<T_{B}(x), I_{B}(x), F_{B}(x)>, v(x)\right) \mid x \in X\right\}$ be two possibility neutrosophic cubic sets in $X$. Then the distance measure of $\mathscr{P}_{X}$ and $\mathscr{Q}_{X}$ be defined as follows:

$$
d\left(\mathscr{P}_{X}, \mathscr{Q}_{X}\right)=\frac{1}{10} \sum_{i=1}^{n}\left[\begin{array}{c}
\left(\left|A_{T}^{-}\left(x_{i}\right)-B_{T}^{-}\left(x_{i}\right)\right|+\left|A_{T}^{+}\left(x_{i}\right)-B_{T}^{+}\left(x_{i}\right)\right|+\left|A_{I}^{-}\left(x_{i}\right)-B_{I}^{-}\left(x_{i}\right)\right|+\left|A_{I}^{+}\left(x_{i}\right)-B_{I}^{+}\left(x_{i}\right)\right|\right. \\
+\left|A_{F}^{-}\left(x_{i}\right)-B_{F}^{-}\left(x_{i}\right)\right|+\left|A_{F}^{+}\left(x_{i}\right)-B_{F}^{+}\left(x_{i}\right)\right|+\left|T_{A}\left(x_{i}\right)-T_{B}\left(x_{i}\right)\right|+\left|I_{A}\left(x_{i}\right)-I_{B}\left(x_{i}\right)\right| \\
\left.+\left|F_{A}\left(x_{i}\right)-F_{B}\left(x_{i}\right)\right|+\left|\mu\left(x_{i}\right)-v\left(x_{i}\right)\right|\right)
\end{array}\right]
$$

Theorem 1. Let $\mathscr{P}_{X}, \mathscr{Q}_{X}, \mathscr{W}_{X}$ be possibility neutrosophic cubic sets. Then

$$
\begin{aligned}
& \text { (1) } d\left(\mathscr{P}_{X}, \mathscr{Q}_{X}\right)=d\left(\mathscr{Q}_{X}, \mathscr{P}_{X}\right) ; \\
& \text { (2) } 0 \leq d\left(\mathscr{P}_{X}, \mathscr{Q}_{X}\right) \leq 1 ; \\
& \text { (3) } d\left(\mathscr{P}_{X}, \mathscr{Q}_{X}\right)=0, \text { iff } \mathscr{P}_{X}=\mathscr{Q}_{X} ; \\
& \text { (4) } d\left(\mathscr{P}_{X}, \mathscr{Q}_{X}\right)+d\left(\mathscr{Q}_{X}, \mathscr{W}_{X}\right) \geq d\left(\mathscr{P}_{X}, \mathscr{W}_{X}\right) .
\end{aligned}
$$

Proof. The proof of theorems (1), (2), (3) are very simple. (4) of Theorem 3.1 is easy to prove by using the properties of inequalities.

Definition 25. Let $X=\left\{x_{1}, x_{2}, \cdots, x_{n}\right\}$ be a universe, $\mathscr{P}_{X}=\left\{\left(x,<\left[A_{T}^{-}(x), A_{T}^{+}(x)\right],\left[A_{I}^{-}(x), A_{I}^{+}(x)\right]\right.\right.$, $\left.\left.\left[A_{F}^{-}(x), A_{F}^{+}(x)\right]>,<T_{A}(x), I_{A}(x), F_{A}(x)>, \mu(x)\right) \mid x \in X\right\}$ and $\mathscr{Q}_{X}=\left\{\left(x,<\left[B_{T}^{-}(x), B_{T}^{+}(x)\right]\right.\right.$, $\left.\left.\left[B_{I}^{-}(x), B_{I}^{+}(x)\right],\left[B_{F}^{-}(x), B_{F}^{+}(x)\right]>,<T_{B}(x), I_{B}(x), F_{B}(x)>, v(x)\right) \mid x \in X\right\}$ be two possibility neutrosophic cubic numbers in $X$. Then the similarity measure of $\mathscr{P}_{X}$ and $\mathscr{Q}_{X}$ is defined as follows:

$$
\begin{aligned}
s\left(\mathscr{P}_{X}, \mathscr{Q}_{X}\right) & =1-d\left(\mathscr{P}_{X}, \mathscr{Q}_{X}\right) \\
& =1-\frac{1}{10} \sum_{i=1}^{n}\left[\begin{array}{c}
\left(\left|A_{T}^{-}\left(x_{i}\right)-B_{T}^{-}\left(x_{i}\right)\right|+\left|A_{T}^{+}\left(x_{i}\right)-B_{T}^{+}\left(x_{i}\right)\right|+\left|A_{I}^{-}\left(x_{i}\right)-B_{I}^{-}\left(x_{i}\right)\right|+\left|A_{I}^{+}\left(x_{i}\right)-B_{I}^{+}\left(x_{i}\right)\right|\right. \\
+\left|A_{F}^{-}\left(x_{i}\right)-B_{F}^{-}\left(x_{i}\right)\right|+\left|A_{F}^{+}\left(x_{i}\right)-B_{F}^{+}\left(x_{i}\right)\right|+\left|T_{A}\left(x_{i}\right)-T_{B}\left(x_{i}\right)\right|+\left|I_{A}\left(x_{i}\right)-I_{B}\left(x_{i}\right)\right| \\
\left.+\left|F_{A}\left(x_{i}\right)-F_{B}\left(x_{i}\right)\right|+\left|\mu\left(x_{i}\right)-v\left(x_{i}\right)\right|\right)
\end{array}\right] .
\end{aligned}
$$

Theorem 2. Let $\mathscr{P}_{X}, \mathscr{Q}_{X}, \mathscr{W}_{X}$ be possibility neutrosophic cubic sets. Then

$$
\begin{aligned}
& \text { (1) } s\left(\mathscr{P}_{X}, \mathscr{Q}_{X}\right)=s\left(\mathscr{Q}_{X}, \mathscr{P}_{X}\right) ; \\
& (2) 0 \leq s\left(\mathscr{P}_{X}, \mathscr{Q}_{X}\right) \leq 1 ; \\
& \text { (3) } s\left(\mathscr{P}_{X}, \mathscr{Q}_{X}\right)=1, \text { iff } \mathscr{P}_{X}=\mathscr{Q}_{X} .
\end{aligned}
$$

Proof. The proof of theorems (1), (2), (3) can be proved directly. 
Example 9. Let $\mathscr{P}_{X}, \mathscr{Q}_{X}, \mathscr{W}_{X}, \mathscr{R}_{X}$ be possibility neutrosophic cubic sets. Suppose that

$$
\begin{gathered}
\mathscr{P}_{X}=\left[\begin{array}{l}
(\langle[0.8,0.9],[0.0,0.1],[0.1,0.2]\rangle,\langle 0.8,0.0,0.1\rangle, 0.8) \\
(\langle[0.6,0.7],[0.1,0.2],[0.2,0.3]\rangle,\langle 0.8,0.1,0.1\rangle, 0.6) \\
(\langle[0.5,0.6],[0.3,0.4],[0.2,0.3]\rangle,\langle 0.5,0.3,0.2\rangle, 0.5)
\end{array}\right] . \\
\mathscr{Q}_{X}=\left[\begin{array}{l}
(\langle[0.6,0.8],[0.1,0.2],[0.0,0.1]\rangle,\langle 0.7,0.1,0.1\rangle, 0.6) \\
(\langle[0.5,0.7],[0.3,0.5],[0.2,0.3]\rangle,\langle 0.7,0.3,0.2\rangle, 0.7) \\
(\langle[0.3,0.5],[0.8,0.9],[0.4,0.6]\rangle,\langle 0.5,0.7,0.4\rangle, 0.6)
\end{array}\right] . \\
\mathscr{W}_{X}=\left[\begin{array}{l}
(\langle[0.7,0.8],[0.0,0.1],[0.0,0.1]\rangle,\langle 0.7,0.0,0.1\rangle, 0.8) \\
(\langle[0.6,0.8],[0.2,0.3],[0.1,0.2]\rangle,\langle 0.7,0.2,0.2\rangle, 0.7) \\
(\langle[0.5,0.7],[0.3,0.4],[0.1,0.3]\rangle,\langle 0.6,0.2,0.1\rangle, 0.7)
\end{array}\right] . \\
\mathscr{R}_{X}=\left[\begin{array}{l}
(\langle[0.6,0.7],[0.1,0.2],[0.1,0.2]\rangle,\langle 0.7,0.1,0.2\rangle, 0.9) \\
(\langle[0.5,0.7],[0.2,0.3],[0.2,0.4]\rangle,\langle 0.7,0.1,0.2\rangle, 0.8) \\
(\langle[0.6,0.8],[0.2,0.4],[0.1,0.3]\rangle,\langle 0.6,0.3,0.2\rangle, 0.7)
\end{array}\right] .
\end{gathered}
$$

From the above definition we can get the following results.

$$
\begin{aligned}
& d\left(\mathscr{P}_{X}, \mathscr{Q}_{X}\right)=0.47, d\left(\mathscr{W}_{X}, \mathscr{R}_{X}\right)=0.21 . \\
& s\left(\mathscr{P}_{X}, \mathscr{Q}_{X}\right)=9.53, s\left(\mathscr{W}_{X}, \mathscr{R}_{X}\right)=9.79 .
\end{aligned}
$$

Combined with the above theoretical knowledge, we present an algorithm to solve the multi-attribute decision problem.

Suppose that $X=\left\{x_{1}, x_{2}, \cdots, x_{n}\right\}$ is an alternative set, and $E=\left\{e_{1}, e_{2}, \cdots, e_{m}\right\}$ is an attribute set. If the evaluation results given by all experts are in the form of possibility neutrosophic cubic sets, the following method can be used to choose the best alternative.

Decision-making algorithm:

step 1: According to the evaluation of experts, the possibility neutrosophic cubic sets is obtained as follows:

$$
\mathscr{P}_{X}=\left\{\left(x,<A_{T}(x), A_{I}(x), A_{F}(x)>,<T(x), I(x), F(x)>, \mu(x)\right) \mid x \in X\right\} .
$$

step 2: The following equality is solved by combining the related definitions of possibility neutrosophic cubic sets and step 1,

$S\left(\mathscr{P}_{X}\right)=\sum_{i=1}^{n}\left[\left(4+A_{T}^{-}\left(x_{i}\right)+A_{T}^{+}\left(x_{i}\right)-A_{I}^{-}\left(x_{i}\right)-A_{I}^{+}\left(x_{i}\right)-A_{F}^{-}\left(x_{i}\right)-A_{F}^{+}\left(x_{i}\right)\right)+\left(2+T\left(x_{i}\right)-I\left(x_{i}\right)-F\left(x_{i}\right)\right)+\mu\left(x_{i}\right)\right] / 10$.

step 3: If $S\left(x_{i}\right) \neq S\left(x_{j}\right)(i \neq j, i, j \in n)$, sort the results of step 2 to get the optimal alternative. Otherwise, perform step 4.

step 4: If $S\left(x_{i}\right)=S\left(x_{j}\right)(i \neq j, i, j \in n)$, then solve the following equality,

$$
A\left(\mathscr{P}_{X}\right)=\sum_{i=1}^{n}\left[A_{T}^{-}\left(x_{i}\right)+A_{T}^{+}\left(x_{i}\right)-A_{F}^{-}\left(x_{i}\right)-A_{F}^{+}\left(x_{i}\right)+\left(T\left(x_{i}\right)-F\left(x_{i}\right)\right)+\mu\left(x_{i}\right)\right] / 4 .
$$

and sort the results of step 4 to get the optimal alternative.

\section{Application and Comparative Analysis}

In this part, we give a specific application of the algorithm and make a qualitative comparison between the proposed method and the existing method to illustrate the superiority of the proposed method. 


\subsection{Application}

According to the algorithm given above, we can know that for any number of alternatives and attributes, we can obtain the optimal alternative by calculating their score functions. It can satisfy both the symmetric structure of $n * n$ and the asymmetric structure of $n * m$. This paper chooses an asymmetric structure without speciality to illustrate the application of the scoring function in the possibility neutrosophic cubic sets. The calculation method of the symmetric structure is the same.

Example 10. Mr. Zhang wants to choose a school for his son and there are four schools $X=\left\{x_{1}, x_{2}, x_{3}, x_{4}\right\}$ for him to choose from. Mr. Zhang analyzes and compares $e_{1}=$ cost, $e_{2}=$ atmosphere, and $e_{3}=$ teachingquality, and obtained a preliminary evaluation. The evaluation value was given in the form of possibility neutrosophic cubic sets. Choose the best school according to Mr. Zhang's evaluation.

step 1: According to the evaluation results, the possibility neutrosophic cubic set is obtained

$$
\begin{aligned}
& \mathscr{P}_{X}\left(x_{1}\right)=\left[\begin{array}{l}
(\langle[0.5,0.6],[0.1,0.3],[0.2,0.4]\rangle,\langle 0.6,0.2,0.3\rangle, 0.5) \\
(\langle[0.5,0.6],[0.1,0.3],[0.2,0.4]\rangle,\langle 0.6,0.2,0.3\rangle, 0.5) \\
(\langle[0.6,0.8],[0.2,0.3],[0.1,0.2]\rangle,\langle 0.7,0.2,0.1\rangle, 0.7)
\end{array}\right], \\
& \mathscr{P}_{X}\left(x_{2}\right)=\left[\begin{array}{l}
(\langle[0.6,0.8],[0.1,0.2],[0.2,0.3]\rangle,\langle 0.7,0.1,0.2\rangle, 0.7) \\
(\langle[0.6,0.7],[0.1,0.2],[0.2,0.3]\rangle,\langle 0.6,0.1,0.2\rangle, 0.6) \\
(\langle[0.6,0.7],[0.3,0.4],[0.1,0.2]\rangle,\langle 0.7,0.4,0.1\rangle, 0.7)
\end{array}\right], \\
& \mathscr{P}_{X}\left(x_{3}\right)=\left[\begin{array}{l}
(\langle[0.4,0.6],[0.2,0.3],[0.1,0.3]\rangle,\langle 0.6,0.2,0.2\rangle, 0.7) \\
(\langle[0.5,0.6],[0.2,0.3],[0.3,0.4]\rangle,\langle 0.6,0.3,0.4\rangle, 0.6) \\
(\langle[0.5,0.7],[0.2,0.3],[0.3,0.4]\rangle,\langle 0.6,0.2,0.3\rangle, 0.6)
\end{array}\right], \\
& \mathscr{P}_{X}\left(x_{4}\right)=\left[\begin{array}{l}
(\langle[0.7,0.8],[0.1,0.2],[0.1,0.2]\rangle,\langle 0.8,0.1,0.2\rangle, 0.8) \\
(\langle[0.6,0.7],[0.1,0.2],[0.1,0.3]\rangle,\langle 0.7,0.1,0.2\rangle, 0.7) \\
(\langle[0.6,0.7],[0.3,0.4],[0.2,0.3]\rangle,\langle 0.7,0.3,0.2\rangle, 0.6)
\end{array}\right] .
\end{aligned}
$$

step 2: Calculate the score functions of all possibility neutrosophic cubic sets;

$$
S\left(x_{1}\right)=2.11, S\left(x_{2}\right)=2.23, S\left(x_{3}\right)=2.01, S\left(x_{4}\right)=2.28 \text {. }
$$

step 3: Ranking the score functions of all alternatives by size, which is the order of the alternative.

$$
S\left(x_{4}\right) \geq S\left(x_{2}\right) \geq S\left(x_{1}\right) \geq S\left(x_{3}\right) .
$$

Hence, the alternative 4 is the best choice. That is to say, according to the assessment, Mr. Zhang should choose the fourth school.

\subsection{Comparative Analysis}

At present, many decisions are multi-attribute decision-making problems, and the choice of decision-making environment will have a certain impact on the results of decision-making. Therefore, it is very important to choose a suitable decision-making environment in multi-attribute decision-making problems. Based on the existing decision environment, this paper proposes a new environment for multi-attribute decision-making. As this paper proposes a new decision environment, it is impossible to make a quantitative analysis and comparison in the comparative analysis. Next, we use the qualitative analysis method and some existing methods for comparative analysis to illustrate the method proposed in this paper.

Firstly, compared with some existing extension sets of fuzzy sets, such as intuitionistic fuzzy sets [13] and interval neutrosophic sets [23], we find that they only study the membership 
degree and non-membership degree of elements, and do not consider the uncertainty of elements. The neutrosophic cubic sets are an extension of the neutrosophic sets and the cubic sets. They consider the membership, non-membership and uncertainty of the elements at the same time, which makes the decision-making factors more comprehensive. In this way, the decision result is more likely to approach the ideal result.

Secondly, compared with the decision-making method of neutrosophic cubic sets [31,34], possibility neutrosophic cubic sets not only consider the membership, non-membership and uncertainty of elements, but also consider the possibility of each element in the set, giving different weights to elements, making the distribution of elements closer to the overall level of the research problem, so as to make the decision result more real.

Finally, a simple linear model is proposed to solve the multi-attribute decision-making problem, which makes the solution more convenient and relatively simple.

\section{Conclusions}

The neutrosophic cubic sets are an extension of the neutrosophic sets and the cubic sets. They also consider the membership, non-membership and uncertainty of the elements in the set, which is more comprehensive than the fuzzy sets and the hesitant fuzzy sets. According to the current research on the neutrosophic cubic sets, this paper considers the possibility of the occurrence of elements in the neutrosophic cubic sets, and puts forward the definition of possibility neutrosophic cubic sets, and the empty sets, the complete sets, the complement sets, distance measure and similarity measure of the possibility neutrosophic cubic sets are defined. At the same time, the definition of the equality, inclusion, R-union and R-intersection of any two possibility neutrosophic cubic sets is given. Under some certain conditions, the R-union and R-intersection of any two possibility internal neutrosophic cubic sets are still possibility internal neutrosophic cubic sets. Finally, the score function is an important indicator in multiple attribute decision making problems; combined with the score function, we give a solution of possibility neutrosophic cubic sets in multiple attribute decision making, and apply this method to concrete numerical examples to illustrate the effectiveness of the score function in solving multiple attribute decision making problems.

The possibility neutrosophic cubic sets consider the membership, non-membership and uncertainty of the elements of the set, and the possibility that the elements belong to the set. Therefore, combined with the advantages of the possibility neutrosophic cubic sets, it can be applied to imperfect systems in actual production to better solve production problems [44]. At the same time, in the possibility neutrosophic cubic sets, we propose a simple linear model to solve multi-attribute decision-making problems. In future research, we can combine the non-linear model method proposed in the concept lattice of multi-adjoint t-concept lattices [45] and word-level neutrosophic sentiment similarity [46] to discuss the application of the non-linear model method in the possibility neutrosophic cubic sets to solve multi-attribute decision-making problems in this environment. It is believed that in the future, the application of the neutrosophic cubic sets in these aspects will be a good research topic, which is worth further research.

Author Contributions: All authors contributed equally. All authors have read and agreed to the published version of the manuscript.

Funding: This work was supported by the National Natural Science Foundation of China (11661053, 11771198), and the Provincial Natural Science Foundation of Jiangxi, China(20181BAB201003).

Acknowledgments: The authors would like to thank the editors and anonymous reviewers for their insightful and constructive commendations that have lead to an improved version of this paper.

Conflicts of Interest: The authors declare no conflict of interest. 


\section{References}

1. Zadeh, L.A. Fuzzy sets. Inf. Control 1965, 8, 338-353. [CrossRef]

2. Nguyen, V.U. Tender evaluation by fuzzy sets. J. Constr. Eng. Manag. 1985, 111, 231-243. [CrossRef]

3. Yager, R.R. Database discovery using fuzzy sets. Int. J. Intell. Syst. 1996, 11, 691-712. [CrossRef]

4. Pal, S.K.; King, R.A. On edge detection of X-ray images using fuzzy sets. IEEE Trans. Pattern Anal. Mach. Intell. 1983, 5, 69-77. [CrossRef]

5. Gurcanli, G.E.; Mungen, U. An occupational safety risk analysis method at construction sites using fuzzy sets. Int. J. Ind. Ergon. 2009, 39, 371-387.

\section{[CrossRef]}

6. Li, H.; Yin, S.; Yang, Y. Some preference relations based on q-rung orthopair fuzzy sets. Int. J. Intell. Syst. 2019, 34, 2920-2936. [CrossRef]

7. Wu, H. Generalized extension principle for non-normal fuzzy sets. Fuzzy Optim. Decis. Mak. 2019, 18, $399-432$. [CrossRef]

8. Verma, R.; Merigó, J. On generalized similarity measures for Pythagorean fuzzy sets and their applications to multiple attribute decisio-making. Int. J. Intell. Syst. 2019, 34, 2556-2583. [CrossRef]

9. Ejegwa, P.A.; Awolola, J.A. Novel distance measures for Pythagorean fuzzy sets with applications to pattern recognition problems. Granular Comput. 2019, 1-9.

10. Atanassov, K.T. Intuitionistic fuzzy sets. Fuzzy Sets Syst. 1986, 20, 87-96. [CrossRef]

11. Atanassov, K.T. Interval valued intuitionistic fuzzy sets. InIntuitionistic Fuzzy Sets 1999, 35, 139-177.

12. Torra, V. Hesitant fuzzy sets. Int. J. Intell. Syst. 2010, 25, 529-539. [CrossRef]

13. Liuab, H.W. Multi-criteria decision-making methods based on intuitionistic fuzzy sets. Eur. J. Oper. Res. 2007, 179, 220-233.

14. De, S.K.; Biswas, R.; Roy, A.R. An application of intuitionistic fuzzy sets in medical diagnosis. Fuzzy Sets Syst. 2001, 117, 209-213. [CrossRef]

15. Zhang, Q.S.; Yao, H.X.; Zhang, Z.H. Some similarity measures of interval-valued intuitionistic fuzzy sets and application to pattern recognition. Appl. Mech. Mater. 2010, 44-47, 3888-3892. [CrossRef]

16. Xu, Z.; Xia, M. Distance and similarity measures for hesitant fuzzy sets. Inf. Sci. 2011, 181, 2128-2138. [CrossRef]

17. Smarandache, F. A unifying field in logics: Neutrosophic logic, neutrosophic set, neutrosophic probability and statistics (fourth edition). Mult.-Valued Log. 2001, 95, 7-108.

18. Smarandache, F. Neutrosophic set-a generalization of the intuitionistic fuzzy set. In Proceedings of the IEEE International Conference on Granular Computing, Atlanta, GA, USA, 10-12 May 2006.

19. Smarandache, F. Single valued neutrosophic sets. Rev. Air Force Acad. 2013, 10, 9-14.

20. Wang, H.B.; Smarandache, F.; Zhang, Y.Q.; Sunderraman, R. Interval neutrosophic sets and logic: Theory and applications in computing. Comput. Sci. 2005, 65, 87.

21. Zhang, H.; Wang, Q.; Chen, X. Interval neutrosophic sets and their application in multicriteria decision making problems. Sci. World J. 2014, 1, 645953. [CrossRef]

22. Broumi, S.; Smarandache, F. New distance and similarity measures of interval neutrosophic sets. In Proceedings of the FUSION 2014-17th International Conference on Information Fusion, Salamanca, Spain, 7-10 July 2014; doi:10.6084/M9.FIGSHARE.1502585. [CrossRef]

23. Zhang, H.Y.; Pu, J.; Wang, J.Q.; Chen, X.H. An improved weighted correlation coefficient based on integrated weight for interval neutrosophic sets and its application in multi-criteria decision-making problems. Int. J. Comput. Intell. Syst. 2015, 8, 1027-1043. [CrossRef]

24. Huang, H. New distance measure of single-valued neutrosophic sets and its application. Int. J. Intell. Syst. 2016, 31, 1021-1032. [CrossRef]

25. Shahzadi, G.; Akram, M.; Saeid, A.B. An application of single-valued neutrosophic sets in medical diagnosis. Neutrosophic Sets Syst. 2017, 18, 80-88.

26. Ye, J. Another form of correlation coefficient between single valued neutrosophic sets and its multiple attribute decision-making method. Neutrosophic Sets Syst. 2013, 1, 8-12.

27. Broumi, S.; Smarandache, F. Correlation coefficient of interval neutrosophic set. Appl. Mech. Mater. 2013, 436, 511-517. [CrossRef] 
28. Chi, P.; Liu, P. An extended TOPSIS method for the multiple attribute decision making problems based on interval neutrosophic set. Neutrosophic Sets Syst. 2013, 1, 63-70.

29. Jun, Y.B.; Kim, C.S.; Yang, K.O. Cubic sets. Ann. fuzzy Math. Inform. 2012, 4, 83-98.

30. Jun, Y.B.; Smarandache, F.; Chang, S.K. Neutrosophic cubic sets. New Math. Nat. Comput. 2016, $13,14$. [CrossRef]

31. Tu, A.; Ye, J.; Bing, W. Multiple attribute decision-making method using similarity measures of neutrosophic cubic sets. Symmetry 2018, 10, 215. [CrossRef]

32. Ye, J. Operations and aggregation method of neutrosophic cubic numbers for multiple attribute decision-making. Soft Comput. 2018, 22, 7435-7444. [CrossRef]

33. Jun, Y.B.; Smarandache, F.; Chang, S.K. R-intersections and R-unions of neutrosophic cubic sets. In Proceedings of the 2016 IEEE International Conference on Fuzzy Systems (FUZZ-IEEE), Vancouver, BC, Canada, 24-29 July 2016; pp. 2441-2443.

34. Xue, H.; Yu, M.; Chen, C. Research on novel correlation coefficient of neutrosophic cubic sets and its applications. Math. Probl. Eng. 2019, 1, 1-10. [CrossRef]

35. Alkhazaleh, S.; Salleh, A.R.; Hassan, N. Possibility fuzzy soft set. Adv. Decis. Sci. 2015, 2011, 1-18. [CrossRef]

36. Bashir, M.; Salleh, A.R.; Alkhazaleh, S. Possibility intuitionistic fuzzy soft set. Acad. J. Appl. Math. Sci. 2012, 3, 1-24. [CrossRef]

37. Yang, Y.; Meng, C.C. On possibility interval-valued fuzzy soft sets. Appl. Mech. Mater. 2013, 336-338, 2288-2302. [CrossRef]

38. Karaaslan, F. Possibility neutrosophic soft sets with applications in decision making and similarity measure. arXiv 2014, arXiv:1407.3211.

39. Karaaslan, F. Possibility neutrosophic soft sets and PNS-decision making method. Appl. Soft Comput. 2016, 54, 403-414. [CrossRef]

40. Hassan, N.; Al-Quran, A. Possibility neutrosophic vague soft expert set for decision under uncertainty. Am. Inst. Phys. Conf. Ser. 2017, 1830, 070007.

41. Dombi, J. A general class of fuzzy operators, the demorgan class of fuzzy operators and fuzziness measures induced by fuzzy operators. Fuzzy Sets Syst. 1982, 8, 149-163. [CrossRef]

42. Ye, J. Linguistic neutrosophic cubic numbers and their multiple attribute decision-making method. Information 2017, 8, 110. [CrossRef]

43. Shi, L.; Ye, J. Dombi aggregation operators of neutrosophic cubic sets formultiple attribute decision-making. Algorithms 2018, 11, 1-15.

44. Bucolo, M.; Buscarino, A.; Famoso, C.; Fortuna, L.; Frasca, M. Control of imperfect dynamical systems. Nonlinear Dyn. 2019, 98, 2989-2999. [CrossRef]

45. Medina, J.; Ojedaaciego, M. Multi-adjoint t-concept lattices. Inf. Sci. 2010, 180, 712-725. [CrossRef]

46. Smarandache, F.; Colhon, M.; Vlăduţescu, Ş.; Negrea, X. Word-level neutrosophic sentiment similarity. Appl. Soft Comput. 2019, 80, 167-176. [CrossRef]

(C) 2020 by the authors. Licensee MDPI, Basel, Switzerland. This article is an open access article distributed under the terms and conditions of the Creative Commons Attribution (CC BY) license (http://creativecommons.org/licenses/by/4.0/). 